(C) 2010

Daniel M Curlik II

ALL RIGHTS RESERVED 


\title{
LEARNING INCREASES THE SURVIVAL OF NEWBORN NEURONS PROVIDED THAT LEARNING IS DIFFICULT TO ACHIEVE AND SUCCESSFUL by DANIEL M CURLIK II
}

\author{
A thesis submitted to the \\ Graduate School-New Brunswick \\ Rutgers, The State University of New Jersey \\ in partial fulfillment of the requirements \\ for the degree of \\ Master of Science \\ Graduate Program in Psychology \\ written under the direction of \\ Dr. Tracey J. Shors, PhD \\ and approved by
}

New Brunswick, New Jersey

May 2010 


\title{
ABSTRACT OF THE THESIS
}

\section{LEARNING INCREASES THE SURVIVAL OF NEWBORN NEURONS PROVIDED THAT LEARNING IS DIFFICULT TO ACHIEVE AND SUCCESSFUL}

\author{
By DANIEL M CURLIK II
}

\author{
Thesis Director: \\ Dr. Tracey J. Shors
}

Processes of learning can increase the survival of new neurons generated in the adult hippocampal formation (Gould et al., 1999; Shors, 2009). However, only some types of learning are effective. Recent studies demonstrate that animals that learn the conditioned response $(\mathrm{CR})$, but require more trials to do so, rescue more new neurons than animals that quickly acquire the CR, or those that fail to acquire the CR. These studies altered task parameters to modify the number of trials required to learn a conditioned response. Here we asked whether pharmacological manipulations that decrease or increase learning would decrease and increase, respectively, the number of cells that remain in the hippocampus after training. To answer this question, we first prevented learning with the competitive NMDA receptor antagonist ( \pm )-3-(2Carboxypiperazin-4-yl)propyl-1-phosphonic acid (CPP). Administration of the NMDA receptor antagonist CPP each day before training prevented acquisition of the trace eyeblink response, and the subsequent increase in neuronal survival. Second, we facilitated learning with the cognitive enhancer d-cycloserine (DCS), a compound that increases NMDA receptor activity via its actions at the glycine binding site. 
Administration of the NMDA receptor partial agonist DCS each day before training increased the number of learned responses and the number of cells that survived. Animals that successfully acquired the CR early in training possessed more cells than those exposed to unpaired stimuli but those that learned later in training retained even more newborn neurons. DCS \& CPP did not alter performance or cell number when administered after training. These results demonstrate that NMDA receptor activation modifies learning and as a consequence, alters the number of surviving neurons in the hippocampus. Moreover, they demonstrate that associative learning increases neuronal survival provided that the learning is both difficult to achieve and successful. 


\section{Acknowledgement}

First and foremost, I thank my advisor, Dr. Tracey J. Shors, for her continued advice and guidance during my thesis work. Additionally, I thank my labmates, Megan Anderson and Lisa Maeng, for their daily assistance and advice. Finally, I thank my family, especially my mother, grandmother, and sister, for their constant support throughout the years. 
Table of contents

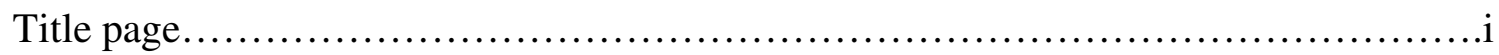

Abstract......................................................................

Acknowledgement..........................................................

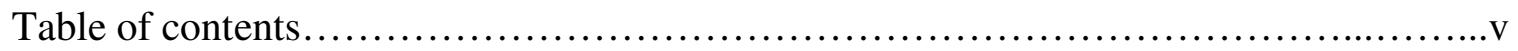

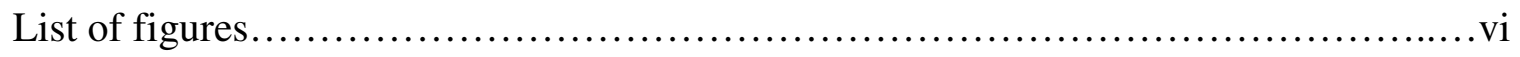

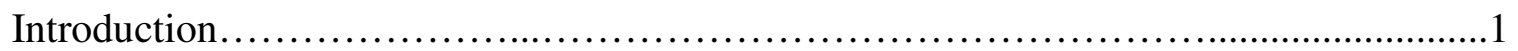

Methods.............................................................................

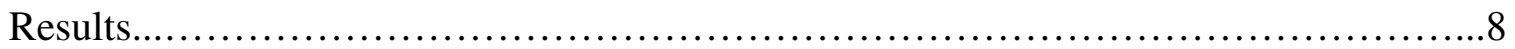

Discussion.................................................................. 16

Figures................................................................. 23

References.................................................................. 31 


\section{List of figures:}

Figure 1. CPP prevents learning and the subsequent increase in neuronal survival...................27

Figure 2. DCS facilitates learning, thereby increasing neuronal survival......................28

Figure 3. Correlations between the percentage of CRs and the number of surviving cells............29

Figure 4. Representative BrdU labeled cells........................................ 30 


\section{Introduction}

The dentate gyrus (DG) of the hippocampus generates new granule neurons throughout life (Altman \& Das 1965; Caviness et al., 1973; Kaplan \& Hinds, 1977; Barnea et al., 1994; Kornack \& Rakic, 1999). It is estimated that the adult hippocampus generates at least five thousand new cells per day (Cameron \& McKay, 2001), the vast majority of which differentiate into neurons (Cameron et al., 1993; Hastings \& Gould, 1999; Markakis \& Gage, 1999); however, over half of these cells die within a few weeks of their birth (Gould et al., 1999). One week after they are born these neurons display a remarkable sensitivity to various forms of learning which can prevent their death, thereby increasing the number of new neurons that survive to become functional neurons in the adult brain (Ambrogini et al., 2000, Dayer et al., 2003, Gould et al., 1999; Leuner et al., 2004; Waddell \& Shors, 2008; Shors, 2009).

The first study to report that learning increases neuronal survival indicated that tasks that depend on the hippocampus are most effective (Gould et al., 1999). These tasks included trace eyeblink conditioning and training on the spatial version of the Morris water maze. In contrast, training on tasks that do not depend on the hippocampus, such as delay eyeblink conditioning or visible cue learning in a maze, did not increase the survival of the new cells (Gould et al., 1999). Since then, it has been determined that hippocampal-dependence, per se, is not necessary to increase survival of these cells (Reviewed in Shors, 2008). For example, animals that were trained with a hippocampalindependent form of eyeblink conditioning, known as contiguous trace conditioning, possessed more new neurons after learning than naïve controls (Dalla et al., 2007). That said, the most effective tasks tend to depend on the hippocampus for learning itself. 
One explanation for this may be that these tasks tend to be more difficult to learn than their non-hippocampal dependent counterparts. Indeed, tasks that are more difficult to learn (i.e. require more trials of training to reach asymptotic performance) are most effective when it comes to increasing cell survival. In other words, animals that learn, but do so after more training trials, rescue more newborn neurons than animals that quickly acquire the same learned response (Dalla et al., 2007; Waddell \& Shors, 2008). Moreover, recent studies indicate that learning, not merely training, is likewise critical (Sisti and Shors, 2007). Thus, animals that are trained but do not learn do not retain any more cells than animals kept in their home cage. Together, these results indicate that animals that fail to acquire a learned response or do so very rapidly will have fewer surviving newborn neurons than animals that learn but require many trials of training to do so. These conclusions were drawn from studies in which task parameters were manipulated in order to alter acquisition and asymptotic performance. If these conclusions can be generalized to other learning processes and conditions, then similar results should occur if learning is manipulated with pharmacological agents. In the current set of studies, we pharmacology manipulated acquisition of the classically conditioned eyeblink response using a $500 \mathrm{~ms}$ trace interval, because this task reliably increases cell survival after learning. In the first experiment, we prevented acquisition with a competitive NMDA receptor antagonist, CPP. In the second experiment, we enhanced acquisition with the partial glycine receptor agonist d-cylcoserine (DCS). The effects of these agents on performance of the CR and the consequent effect on cell number were determined. 


\section{Methods}

General Methods for all Experiments:

Subjects: Adult male Sprague-Dawley rats between 60 and 90 days of age were individually housed and given access to food and water ad libitum. The animals were maintained on a twelve hour light/dark schedule, with lights on at 7:00 AM. Rats were handled by the experimenter at least one week prior to surgery. Experiments were designed to fully comply with the rules and regulations set forth by the PHS Policy on Humane Care and use of Laboratory Animals and the Guide for the Care and Use of Laboratory Animals.

Surgical Procedures: Animals were anesthetized with sodium pentobarbital $(15 \mathrm{mg} / \mathrm{kg})$ and supplemented with isoflourane gas. They then underwent stereotaxic surgery to implant four perioribital electrodes in the muscles of the right eyelid. The electrodes were composed of a stainless steel wire (0.005 in) that was inserted through the eyelid, under the skin of the scalp, to a headstage that that was mounted onto the skull. Dental cement secured the headstage to four small screws that were partially imbedded within the skull (Servatius and Shors, 1996). Animals were given a minimum recovery period of five days before receiving one single BrdU injection $(200 \mathrm{mg} / \mathrm{kg})$.

Classical Eyeblink Conditioning: Six days after BrdU-injection animals were habituated to the conditioning chambers for one hour while spontaneous blinks were recorded. The 
next day (one week after the BrdU injection), groups of animals began training with either paired trace or explicitly unpaired eyeblink conditioning. Training continued each day for four days. Rats were injected either with the drug or saline either before or after training, depending on the experimental condition. The rats were trained with 200 trials a day. Each trial of trace conditioning consisted of an $82 \mathrm{~dB}$ white noise conditioned stimulus (CS), followed by a 500ms trace interval, followed by a $100 \mathrm{~ms}$ unconditioned stimulus (US) of a $.65 \mathrm{~mA}$ perioribital shock to the right eyelid. The intertrial interval was $25 \pm 5$ seconds. Explicitly unpaired training consisted of the same number of CS and US presentations as trace conditioning except that the CS and the US were presented in an explicitly unpaired manner.

The occurrence of an eyeblink was determined from electromyographic (EMG) recordings of the eyelid muscle. During trace conditioning a baseline recording was taken $250 \mathrm{~ms}$ before every trial. A CR was counted on that trial if a response occurred during the 500ms trace interval, and if that response had an amplitude greater than the maximum amplitude from the baseline recording for that trial, plus four times the baseline's standard deviation. A similar baseline recoding was taken $250 \mathrm{~ms}$ before the onset of the CS during unpaired training. CR's during unpaired training were measured as responses that occurred up to $500 \mathrm{~ms}$ after the onset of the CS. Trials were blocked into sessions of one-hundred consecutive trials. The first 100 trials are also presented in blocks of twenty trials. The number of trials required to reach a behavioral criterion of $60 \%$ CR's during any one session was determined. Animals that reached that criterion were considered to have learned the $\mathrm{CR}$. 
Immunohistochemistry for BrdU: Twenty-one days after BrdU injection animals were deeply anesthetized with sodium pentobarbital $(100 \mathrm{mg} / \mathrm{kg})$ and transcardially perfused with $4 \%$ paraformaldehyde. Brains were extracted and post-fixed in $4 \%$ paraformaldehyde for a minimum of 48 hours before being sectioned. Coronal sections $(40 \mathrm{uM})$ were cut through the entire rostral-caudal extent of the dentate gyrus of one hemisphere with an oscillating tissue slicer. A 1:12 series of slices was mounted onto glass slides for BrdU immunohistochemistry. To stain for BrdU, tissue was heated in $0.1 \mathrm{M}$ citric acid ( $\mathrm{pH} 6.0$ ), incubated in trypsin followed by $2 \mathrm{~N} \mathrm{HCl}$, and then incubated overnight in primary anti-mouse BrdU (1:200) and 0.5\% Tween 20. The next day tissue was incubated in biotinylated anti-mouse antibody (1:200), followed by avidin-biotinhorseradish peroxidase (1:100). Slices were then placed in diaminobenzidine for four minutes. Slides were rinsed in phosphate buffer, counterstained with cresyl violet, and cover-slipped. All slides were coded and the experimenter was blind to the experimental condition. The number of BrdU labeled cells throughout the entire dentate gyrus of every tewelth section was assessed. To accurately estimate the number of BrdU labeled cells throughout the entire hippocampus the number of BrdU labeled cells in the slices was multiplied by twenty-four.

Statistical Analysis: Performance during classical eyeblink conditioning was analyzed with repeated measures analysis of variance (ANOVA) with trial blocks as the repeated measure and number of conditioned responses as the dependent measure. Post-hoc comparisons were made between groups using Tukey's HSD test. BrdU labeled cell 
counts were analyzed with one-way and two-way ANOVAs. Independent samples t-tests and Mann-Whitney U tests were performed as needed.

\section{Experiment 1: Does the NMDA receptor dependent blockade of learning prevent the} increase in cell survival?

The competitive NMDA receptor antagonist ( \pm )-3-(2-Carboxypiperazin-4yl)propyl-1-phosphonic acid (CPP) was dissolved in 0.9\% saline and injected i.p. $(10 \mathrm{mg} / \mathrm{kg})$ each day, one hour prior to training. Previous research has demonstrated that this dose of CPP prevents acquisition of trace eyeblink conditioning (Leuner, Falduto \& Shors 2003). The experiment consisted of seven groups. Group one received CPP injections each day, one hour prior to training with trace eyeblink conditioning (7-10 days after the BrdU injection and labeling of the newly generated cells).Group two received saline injections at the same time point, before trace conditioning. Group 3 received injections of CPP one hour prior to explicitly unpaired conditioning, while Group 4 received saline injections one hour before unpaired training. Group 5 received injections of CPP every day, six hours after training, when CPP administration did not interfere with acquisition of the conditioned response. Group 6 received saline injections every day, six hours after training. The remaining group, Group 7 received daily saline injections at the same time as groups $5 \& 6$; however, this group was not trained with any form of eyeblink conditioning. Comparison of the number of BrdU labeled cells between the first four groups (Groups 1-4) allowed us to determine whether pretraining administration of CPP prevented trace eyeblink conditioning, and the subsequent increase 
in neuronal survival that is normally seen after learning. Comparing the cell counts between the two unpaired groups also allowed us to determine whether CPP administration itself increased neuronal survival. Groups 5-7 served as control groups, which were used to determine whether administration of CPP decreased neuronal survival irrespective of its effects on learning.

Experiment 2: Does the NMDA receptor modulator D-cycloserine increase learning and thereby increase the number of cells that survive?

D-cycloserine (DCS), a partial agonist of the strychnine-insensitive glycine binding site, was used to facilitate acquisition of the CR during trace eyeblink conditioning. The experiment consisted of five groups. Group one received intraperitoneal (i.p.) injections of DCS dissolved in $0.9 \%$ saline $(15 \mathrm{mg} / \mathrm{kg})$ thirty minutes before each day of training with trace conditioning. This dose of d-cycloserine has previously been shown to facilitate learning of trace eyeblink conditioning (Thompson, Moskal \& Disterhoft 1992; Thompson \& Disterhoft, 1997; Waddell, Mallimo \& Shors 2010). Group two received daily injections of saline thirty minutes before trace conditioning. Comparisons between these two groups allowed us to determine whether DCS facilitated acquisition of the trace eyeblink response, and if so, if that facilitated learning resulted in an increase in the number of surviving newborn neurons. Two additional groups (Groups $3 \& 4$ ) received DCS or saline thirty minutes prior to training with explicitly unpaired stimuli. These two groups were examined to determine whether administration of DCS itself might increase neuronal survival, irrespective of its effects on learning. A fifth group of animals received daily injections of DCS six hours after trace conditioning, a time point when DCS administration did not alter acquisition of the 
conditioned response. Comparison of BrdU-labeled cell counts between this group, and group one, which received saline before trace conditioning, allowed us to determine whether DCS decreased neuronal survival, irrespective of its effects on learning.

\section{$\underline{\text { Results }}$}

Experiment 1 Results: Administration of an NMDA receptor antagonist before but not after training prevents learning and the enhancement in neuronal survival seen after learning

Administration of the NMDA receptor antagonist CPP before training each day completely prevented acquisition of the CR. The drug condition (CPP vs saline) and training protocol (paired vs unpaired) were used as independent measures, along with training blocks (100 trial blocks, two blocks per day) as the repeated measure, and the percentage of CRs as the dependent measure. There was an interaction between drug condition and training protocol $\left[F_{(1,20)}=38.71, \mathrm{p} \leq 0.01\right]$, indicating that CPP only altered conditioned responding when it was administered before trace, but not unpaired, conditioning. This was expected because animals trained with unpaired stimuli did not emit CRs. A significant interaction was found between training blocks and drug condition $\left[F_{(7,140)}=2.40, \mathrm{p} \leq 0.05\right]$, revealing that CPP prevented any increase in conditioned responding during training. An interaction was also found between training blocks and training protocol $\left[F_{(7,140)}=3.33, \mathrm{p} \leq 0.01\right]$; therefore, as expected, conditioned responding did not increase in the groups trained with explicitly unpaired conditioning (Fig 1A). 
We next assessed responding during the last day of training. A Two-Way ANOVA was performed with drug condition (CPP vs saline) and training protocol (paired vs unpaired) as the independent measures, and the percentage of CRs emitted during the last session as the dependent measure. Results revealed a significant effect of drug condition $\left[F_{(1,23)}=20.93, \mathrm{p} \leq 0.01\right]$, and training protocol $\left[F_{(1,23)}=31.73, \mathrm{p} \leq 0.01\right]$, with an interaction between drug condition and training protocol $\left[F_{(1,23)}=14.89, \mathrm{p} \leq 0.01\right]$. Therefore, as expected, animals that received saline before trace conditioning emitted a greater percentage of CRs during the last day of training than either of the two groups that received unpaired conditioning, or the group that received CPP before trace conditioning. Furthermore, the percentage of CRs emitted by the group that received CPP before trace conditioning was not different those of the two unpaired groups. Together, these results indicate that administration of CPP before trace eyeblink conditioning completely prevented acquisition of the CR. Furthermore, CPP had no effect on responding to explicitly unpaired stimuli.

Nearly all (86\%) of the animals that received saline during trace conditioning reached criterion (Learned/Saline), whereas none $(0 \%)$ of the animals that were trained with unpaired stimuli (Unpaired/Saline; Unpaired/CPP), or those that received CPP before trace conditioning did (Did Not Learn/CPP). In order to examine the difference in neuronal survival between animals that learned, versus those that did not, a Two-Way ANOVA was conducted, with drug condition and training protocol as the independent measures and the number of BrdU labeled cells as the dependent measure. Only saline treated animals that reached criterion were included in this analysis. Results found a significant interaction between drug condition and training protocol $\left[F_{(1,22)}=8.24\right.$, 
$\mathrm{p} \leq 0.05]$, indicating that only the animals that received saline before trace conditioning (that reached criterion) displayed an increase in neuronal survival (Fig 1B). Significant main effects of drug condition $\left[F_{(1,22)}=23.06, \mathrm{p} \leq 0.01\right]$, and training protocol $\left[F_{(1,22)}=\right.$ $8.62, \mathrm{p} \leq 0.01]$, were also found, revealing that all animals that were treated with CPP, and those that received unpaired training, had no increase in neuronal survival.

To examine the possibility that CPP may decrease neuronal survival irrespective of its effects on learning we examined the number of new cells in two additional groups that received saline (Saline/Control) or CPP (CPP/Control) six hours after training, at a time point when CPP administration did not interfere with acquisition of the conditioned response. A repeated measures ANOVA was conducted, with drug condition as the independent measure, trials blocks (100 trial blocks, two blocks per day) as the repeated measure, and percentage of CRs as the dependent measure. The results revealed a significant main effect of session $\left[F_{(7,98)}=23.35, \mathrm{p} \leq 0.01\right]$, indicating that the percentage of CRs emitted by these two groups increased over the course of training. There was no effect of drug condition $\left[F_{(1,14)}=1.00, \mathrm{p}>0.05\right]$, and no interaction between drug condition and session $\left[F_{(7,98)}=.22, \mathrm{p}>0.05\right]$. Therefore, administration of the NMDA receptor antagonist six hours after every day of trace conditioning did not prevent acquisition of the conditioned response (Fig. 1C). Furthermore, administration of CPP six hours after training did not alter responding on the last session of training $\left(\mathrm{t}_{(14)}=1.082\right.$, $\mathrm{p}>.05$, Saline/Control vs. CPP/Control), nor did it change the number of trials required to reach our criterion of $60 \%$ conditioned responses in any one session $(\mathrm{U}=9.0, \mathrm{p}>.05$, Saline/Control vs. CPP/Control). 
An ANOVA was used to compare the number of BrdU labeled cells in animals that received saline or CPP after training to those in experimentally naïve animals, that received saline injections, but no training (Naïve). There was a difference in cell number among these groups $\left[F_{(2,17)}=7.12, \mathrm{p} \leq 0.01\right]$. Post-hoc Tukey comparisons indicated that animals that reached criterion despite being injected with saline or CPP each day after training possessed more BrdU labeled cells that the naïve controls ( $\mathrm{p}^{\prime} \mathrm{s} \leq 0.05$ ). Thus, animals could still acquire the $\mathrm{CR}$ as long as the antagonist was given after training. In fact, their performance was no different than animals injected with saline and trained with paired stimuli. Since their cell numbers were not different from those that were injected with saline and trained with paired stimuli, we can conclude that antagonism of NMDA receptors before, but not six hours after training, blocks learning of the $\mathrm{CR}$ and thereby prevents the increase in cell survival. In other words, the effect of CPP on cell survival is via its effect on performance of the CR and not an effect of the drug alone on cell survival (Fig 1D).

\section{Experiment 2 Results: $D$-cycloserine facilitates trace conditioning and neurogenesis}

Pretraining administration of D-cycloserine has been previously demonstrated to facilitate learning of trace eyeblink conditioning (Thompson, Moskal \& Disterhoft 1992; Thompson \& Disterhoft, 1997; Waddell, Mallimo \& Shors 2010). The current experiment was conducted to determine whether D-cycloserine facilitated learning would result in an increase in the number of surviving newborn hippocampal neurons. The experiment consisted of five groups. Groups $1 \& 2$ received DCS or saline before training with trace 
eyeblink conditioning. Groups $3 \& 4$ received DCS or saline before training with explicitly unpaired stimuli. Group 5 received DCS six hours after training with trace eyeblink conditioning. To determine whether DCS facilitated acquisition, we examined early acquisition, which was defined as the percentage of CRs emitted during the first session of training. An independent samples t-test comparing the percentage of CRs emitted during the first day of training between the groups that received saline and DCS before trace conditioning indicated that learning in the presence of DCS significantly enhanced early acquisition of the conditioned response $(\mathrm{t}(14)=2.63, \mathrm{p} \leq .05)$. A separate independent samples t-test revealed that DCS had no effect on early acquisition of explicitly unpaired stimuli $(\mathrm{t}(10)=0.70, \mathrm{p}>0.05)$. Therefore, pretraining administration of DCS before trace, but not unpaired, conditioning facilitated early acquisition of the CR.

We next examined responding over the entire 800 trials of training with a repeated measures ANOVA, with trial blocks (100 trial blocks, 2 blocks per day) as the repeated measure, group (Groups 1-5) as the independent measure, and percentage of CRs as the dependent measure. The total number of CRs emitted during training differed between the five groups $\left[F_{(4,28)}=25.47, \mathrm{p} \leq 0.05\right]$. Post-hoc analysis indicated that pretraining administration of DCS did not alter the total number of CRs emitted during the four days of trace conditioning, (p>0.05 Trace vs. DCS During Trace, Fig. 2A). Therefore, while administration of DCS before trace conditioning facilitated acquisition of the $\mathrm{CR}$ on the first day of training, it had no effect on overall responding during the entire four days of training. Additionally, DCS had no effect on the total number of CRs emitted when it was administered six hours after training ( $p>0.05$, Trace vs. DCS 
Control, data not shown), or when it was given before training with explicitly unpaired stimuli ( $\mathrm{p}>0.05$, Unpaired vs DCS unpaired).

Groups that were trained with trace conditioning (Trace, DCS/Trace, DCS Control) increased the percentage of CRs that they emitted across sessions $\left[F_{(7,126)}=\right.$ $20.144, \mathrm{p} \leq 0.05]$, with no interaction between group and session $\left[F_{(14,126)}=1.25, \mathrm{p}>0.05\right]$. Therefore, all trace conditioned groups successfully acquired the conditioned response over the course of training. There was no such increase in responding in animals exposed to unpaired stimuli $\left[F_{(7,70)}=1.20, \mathrm{p}>0.05\right]$ and no difference in overall responding between the two unpaired groups $\left[F_{(1,10)}=0.50, \mathrm{p}>0.05\right]$. Thus, only animals that were trained with trace conditioning successfully acquired the CR. DCS did not alter the number of responses emitted during exposure to explicitly unpaired stimuli. These results would suggest that DCS does not increase conditioning simply by increasing nonspecific responding to the CS.

We next examined the average number of CRs emitted during the last day of training. There were no differences in responding among those groups exposed to trace paired stimuli $\left[F_{(2,18)}=0.82, \mathrm{p}>0.05\right]$. Together, these results indicate that the effects of D-cycloserine on trace eyeblink conditioning are limited to enhancing early acquisition of the CR. Moreover, DCS only facilitated acquisition when it was administered thirty minutes before, but not six hours after, training.

Most (78\%) animals given saline during trace conditioning reached criterion (Learned/Saline), whereas all (100\%) animals given DCS during (Learned/DCS) or after 
trace conditioning (DCS Control) reached criterion. None $(0 \%)$ of the animals that received unpaired conditioning reached criterion.

The number of BrdU labeled cells in the entire dentate gyrus was different between the trained groups $\left[F_{(5,27)}=13.27, \mathrm{p} \leq 0.01\right]$. Post-hoc comparisons revealed that saline treated animals that learned the CR retained more newborn neurons than saline treated animals that did not learn the conditioned response $(\mathrm{p} \leq 0.05$, Learned/Saline vs. Did Not Learn/Saline). The animals that learned the CR also retained more cells than animals that received saline during unpaired training $(\mathrm{p} \leq 0.05$, Learned/Saline vs. Unpaired/Saline), and DCS during unpaired training ( $\mathrm{p} \leq 0.05$, Learned/Saline vs. Unpaired/DCS). Furthermore, when we examined the data from all animals (including those that did not reach criterion) we observed strong positive correlation between the percentage of CRs an individual animal emitted during all 800 trials of training and the number of BrdU labeled cells in that animal's dentate gyrus $(r=.59, p \leq 0.01)$. The percentage of CRs emitted during all 800 trials of training also correlated with the number of new neurons in just the granule cell layer of the dentate gyrus $(r=.61, p \leq 0.01$; Fig. 3A). These data indicate that animals that successfully acquired the conditioned response retained more neurons than those that did not learn the task. Thus, as we have observed in the past, successful acquisition of trace eyeblink conditioning is necessary to increase the number of surviving newborn neurons in the adult dentate gyrus.

Administration of DCS by itself did not increase the survival of newborn neurons ( $>0.05$, Unpaired/DCS vs. Unpaired/Saline). Interestingly, while animals that received DCS during training expressed more CRs at the beginning of training, they did not retain more new neurons; their cell counts were no different from those present in the 
hippocampus of animals that learned well in the absence of the drug $(p>0.05$,

Learned/DCS vs. Learned/Saline, Figure 2B). The cell counts from those animals that received DCS six hours after training, at a time point when it did not facilitate acquisition of the CR, were no different from those of animals that learned in the absence of the drug ( $>$ $>0.05$, DCS Control vs. Learned/Saline, data not shown), indicating that administration of DCS itself did not decrease the survival of newborn neurons.

Previously our laboratory has reported a significant correlation between the number of trials an individual animal requires to reach criterion and the number of BrdUlabeled cells in that animal's dentate gyrus (Waddell \& Shors 2008). A near identical correlation was evident in the present study (Rho $=.59, \mathrm{p} \leq 0.01$, Figure $2 \mathrm{C}$ ).

Furthermore, when we examined the data from only animals that successfully learned the task (i.e. those that reached criterion) we found a strong negative correlation between the percentage of CRs an individual animal emitted during the 800 trials of trace conditioning and the number of surviving newborn neurons in the granule cell layer of that animal's dentate gyrus $(r=-.47, \mathrm{p} \leq 0.05$; Figure 3B). However, there was not a significant correlation between the percentage of CRs an individual animal emitted during the 800 trials of training and the number of surviving neurons in that animal's entire dentate gyrus $(r=-.42, p=0.071$; data not shown $)$. Thus, animals that successfully acquired the conditioned response, and required more trials to do so, retained more of the newborn cells. 


\section{Discussion}

It is now well established that training with various learning tasks can increase the number of newly born cells that survive to become neurons in the adult hippocampus (Shors, 2009). The goal of these experiments was to assess the role of learning, per se in this phenomenon. To do this, we manipulated performance of a learned response in two ways and indeed directions. First, we completely prevented learning from occurring by using an NMDA receptor antagonist - which was given each day before training with trace conditioning. These animals did not learn and they did not retain any more cells in their hippocampus than animals that were trained with unpaired stimuli which also did not learn the conditioned response. These effects were not due to any adverse consequences of the drug itself because animals that were injected with the antagonist after training were able to learn the CR and retained as many new cells as the animals that were trained in the presence of saline. As noted, animals that learned the conditioned response retained many more new cells than animals trained with unpaired stimuli. Thus, preventing learning with an NMDA receptor antagonist prevents the increase in cell number that typically occurs after training. These effects are clear and unambiguous. They are not necessarily surprising because our previous studies have suggested that the effects of training on cell survival only occur in animals that actually learn (Sisti et al., 2007; Dalla et al., 2007). However, these results extend our findings to demonstrate that pharmacological manipulations of learning will have consequences for how many cells ultimately survive to become neurons. Because the cells that are rescued from death by learning remain in the hippocampus for months, at least (Leuner et al., 2004), these 
pharmacological effects of CPP presumably have similarly persistent effects on the circuitry of the adult hippocampus.

In the second experiment, we manipulated learning in the opposite way, again using a drug which affects neuronal activity at the NMDA receptor. The drug, Dcycloserine is a partial glycine receptor agonist which facilitates NMDA receptor mediated excitatory transmission (Hood et al., 1989; Henderson et al., 1990; Rouaud \& Billard 2003), and processes related to learning (Thompson et al., 1992; Thompson \& Disterhoft, 1997; Ledgerwood et al, 2003 Waddell et al., 2010). In this study, we also observed an increase in performance in response to the drug. As a consequence, we report that the number of cells that were retained in the hippocampus in the group that received D-cycloserine before training, which learned well, was elevated when compared to the number in animals that received unpaired training in the presence of the drug. The drug did not increase cell number beyond the numbers observed in animals that were trained and learned after an injection of saline. Furthermore, those animals that naturally failed to acquire the conditioned response did not retain any more of the new cells than those trained with unpaired stimuli. These data suggest that the overall increase in performance in the presence of the drug was not sufficient to increase the number of surviving cells beyond what learning without the drug would accomplish. Again this may not be so surprising. In a previous study we found that learning, when it occurred, rescued nearly all, if not all, of the cells that were available to be rescued (Waddell \& Shors, 2008). One might surmise that there are simply no more new cells to rescue - at least not of this particular cohort. Despite a potential ceiling on the number of cells that can be rescued, animals that were trained in the presence of DCS tended to learn better and more of them 
did so. Thus, one might propose that the drug will increase the number of animals (including humans) that eventually learn and the effect of that overall enhancement of learning would positively influence the number of cells that survive in the hippocampus. Such an interpretation is consistent with recent findings indicating that pre-training administration of D-cycloserine facilitated declarative learning in humans (Onur et al., 2010).

It is noted that exposure to d-cycloserine alone did not seem to affect cell number. Animals that were injected with DCS before unpaired training did not possess any more or fewer cells than those injected with saline and exposed to unpaired training. Moreover, animals injected with DCS after paired training did learn and retained as many cells as those trained with trace conditioning after an injection of saline. Because DCS is known to enhance excitability through facilitating activation of NMDA receptors, these data suggest that this activation is not sufficient to increase cell survival.

The most intriguing results related to individual differences in learning and the number of surviving neurons. When the percentage of learned responses that an individual emitted was correlated with the number of surviving cells, there was a strong and positive relationship. Thus, animals that tended to learn better (i.e., emitted more CRs) also tended to retain more of the new cells in their hippocampus. Since the animals were sacrificed three weeks after training, these cells would be mature neurons. This relationship between learning and neurogenesis can be further parceled into those animals that learned quickly versus those that did not learn quickly, but still did learn. In this case, we only examined animals that reached a criterion of $60 \% \mathrm{CRs}$ in at least one session of training. This criterion is an established one in eyeblink conditioning because it 
tends to capture the vast majority of animals that will learn regardless of how many trials are given. Using this criterion, we correlated the number of trials an individual animal required to reach criterion with the number of BrdU-labeled cells. Again, there was a strong positive correlation. However, in this analysis, the animals that learned but required more trials to reach the learning criterion tended to retain more of the new cells. Furthermore, when we only examined animals that successfully reached criterion, we observed a significant negative correlation between the percentage of CRs an animal emitted over the entire course of training, and the number of surviving newborn neurons. Thus, it would appear that learning well increases the number of surviving cells in the hippocampus but taking longer to do so (or more trials to do so) further increases the likelihood that the new cells will survive.

These results further support work from our laboratory demonstrating that animals that learn, but take longer to do so, retain more newborn neurons in their hippocampus. However, unlike our previous studies examining this effect, in which we manipulated the task parameters (Waddell and Shors, 2008), we now report that facilitation of acquisition can result in a decreased number of surviving newborn neurons. However, there are other possibilities for the observed findings. One injection of BrdU provides only a snapshot of one population of cells -- those that were dividing at the time of, or shortly after, the BrdU injection. It is possible that animals that rapidly acquire the conditioned response, which possess fewer BrdU labeled cells, may actually be rescuing cells that are slightly younger, or slightly older, than the population labeled with BrdU. Although this is a possibility, it does seem unlikely. We and others have found that there is a critical period during which the new cells can be rescued. Cells that are less than 3 days of age or 
greater than 3 weeks were not retained after learning (Epp et al., 2007; Anderson and Shors, 2010). However, finer time points were not examined.

During training of the classically conditioned eyeblink response, cells in area CA1 of the hippocampus become more excitable. This effect is most prevalent in animals that successfully learned the conditioned response (Moyer et al, 1996). During trace fear conditioning, granule cells in the dentate also increase their responsiveness to the CS (Gilmartin \& McEchron, 2005). Both of these effects do not appear to persist beyond the learning phase. Thus, one might propose that an increase in cell excitability in either CA1 or the dentate gyrus contributes to the increase in cell number. In other words, animals that require more trials (and time) to learn the CR would be exposed to a longer period of excitability when compared to the amount in animals that quickly learn the task, or animals that fail to learn. How an increase in excitability would modulate neuronal survival is currently unknown, although one possibility may be through the activation of NMDA receptors in the hippocampal network.

As demonstrated here, NMDA transmission plays a key role in learning and memory. Additionally, it has been known for quite some time that glutamatergic signaling can influence the process of neurogenesis. For example, administration of NMDA receptor antagonists increases the proliferation of adult born neuronal progenitor cells (Cameron et al, 1995; Nacher et al, 2001). Blockade of NMDA receptor activation also decreases the number of surviving neurons in the developing dentate gyrus (Gould et al., 1994). However, very few studies have directly examined the effects of glutamatergic transmission on the survival of adult born neurons. One recent study has reported the genetic deletion of the NR1 subunit of the NMDA receptor, targeting only newborn 
granule neurons, resulted in a decreased survival of 2-3 week old cells; however, no change was observed in the survival of one week old neurons (Tashiro et al, 2006). Such results indicate that direct activation of the NMDA receptor is likely not mediating the survival of newborn neurons in our experiment, as these cells are 7-10 days old during training. They are also consistent with our results since we observed no effect of the drugs alone on cells that were 7-10 days of age. Thus, the effects that we report here are via the effects of the drugs on learning and not likely via their effects on the cells themselves.

From the present and previous data, it is clear that DCS facilitates acquisition of the trace eyeblink response without eliciting any non-specific behavioral sensitization effects (Thompson et al, 1992). Although this compound has been around for decades, it has only recently become the subject of great interest for use in humans. Recent studies indicate that administration of D-cycloserine before exposure therapy in humans with social anxiety disorder resulted in a decrease in some of the symptoms associated with the disorder (Hofmann et al, 2006). Based on this and animal studies, it is proposed that the drug is effective because it increases the learning that occurs during the extinction process (Davis et al, 2006). In another study, we found that DCS not only increases learning but reversed learning deficits induced by stressful experience (Waddell et al., 2010). Additionally, a recent clinical trial has found that D-cycloserine facilitates declarative learning in humans (Onur et al., 2010).

Here we find that D-cycloserine facilitates performance and thereby increases the number of surviving newborn neurons. However, animals that learned very rapidly retained fewer cells than those that learned over more trials. It is noted that we only 
assessed one cohort of cells and thus it is possible that cells that were born at other times were indeed rescued. With that caveat in mind, however, it does appear that rapid learning may not have as demonstrable effects on neurogenesis as does slower learning. Regardless, the present findings indicate that learning will induce the survival of new neurons when that learning is both sufficiently difficult to achieve and successful. 
$\underline{\text { Figure } 1}$

(A) Administration of the NMDA receptor antagonist CPP during trace eyeblink conditioning (CPP During Trace) prevented acquisition of the conditioned response. CPP had no effect on unconditioned responding (CPP Unpaired). Additional animals received saline during training with trace eyeblink conditioning (Trace) or explicitly unpaired stimuli (Unpaired). (B) Animals that received saline during training with trace conditioning that reached criterion (Good Learners) retained more BrdU labeled cells than animals that received CPP during trace conditioning, none of which reached criterion (Did Not Learn/CPP). These Good Learners also retained more new neurons than animals that received saline during unpaired training (Unpaired/Saline), and CPP during unpaired training (Unpaired/CPP). Cell counts from animals that received CPP during trace conditioning were not different from counts of animals that received saline or CPP during unpaired training. (C) CPP had no effect on acquisition or asymptotic performance of trace eyeblink conditioned when it was administered 6hrs after training. (D) CPP had no effect on neuronal survival when administered 6hrs after training, as animals that received saline or CPP after training retained more newborn neurons than experimentally naïve animals. 
$\underline{\text { Figure } 2}$

(A) Intraperitoneal administration of D-cycloserine (DCS) before training with trace eyeblink conditioning (DCS During Trace) facilitated acquisition of the conditioned response, as evidenced by an increase in conditioned responding during the first day of training. DCS had no effect on responding to explicitly unpaired stimuli (DCS Unpaired). For comparison, animals received saline during training with trace conditioning (Trace) or explicitly unpaired stimuli (Unpaired). (B) Animals treated with saline during trace conditioning that reached criterion (Learned/Saline) retained more newborn neurons than animals that received saline during unpaired training (Unpaired/Saline), as well as animals that received DCS during unpaired training (Unpaired/DCS). Those animals that received DCS during trace conditioning (Learned/DCS) retained significantly more newborn neurons than animals that received DCS before unpaired training (Unpaired/DCS). (C) A significant positive correlation was observed between the number of trials an individual animal required to reach criterion and the number of BrdU labeled cells in that animal's Dentate Gyrus. Only animals that successfully reached criterion were included in this analysis. 
$\underline{\text { Figure } 3}$

(A) When the data from all animals in experiment two was examined a significant positive correlation was observed between the percentage of CRs an individual animal emitted over the entire course of training (800 trials) and the number BrdU labeled cells in the granule cell layer of that animal's dentate gyrus. (B) When only the data from animals that reached criterion was analyzed a significant negative correlation was observed between the percentage of CRs an individual animal emitted during training and the number of BrdU labeled cells in the granule cell layer of that animal's dentate gyrus. 
$\underline{\text { Figure } 4}$

Representative BrdU labeled cells from the (A) Learned/Saline (B)

Unpaired/Saline (C) Learned/DCS and (D) Did Not Learn/CPP groups. Arrows indicate BrdU labeled cells. 
$\underline{\text { Figure } 1}$
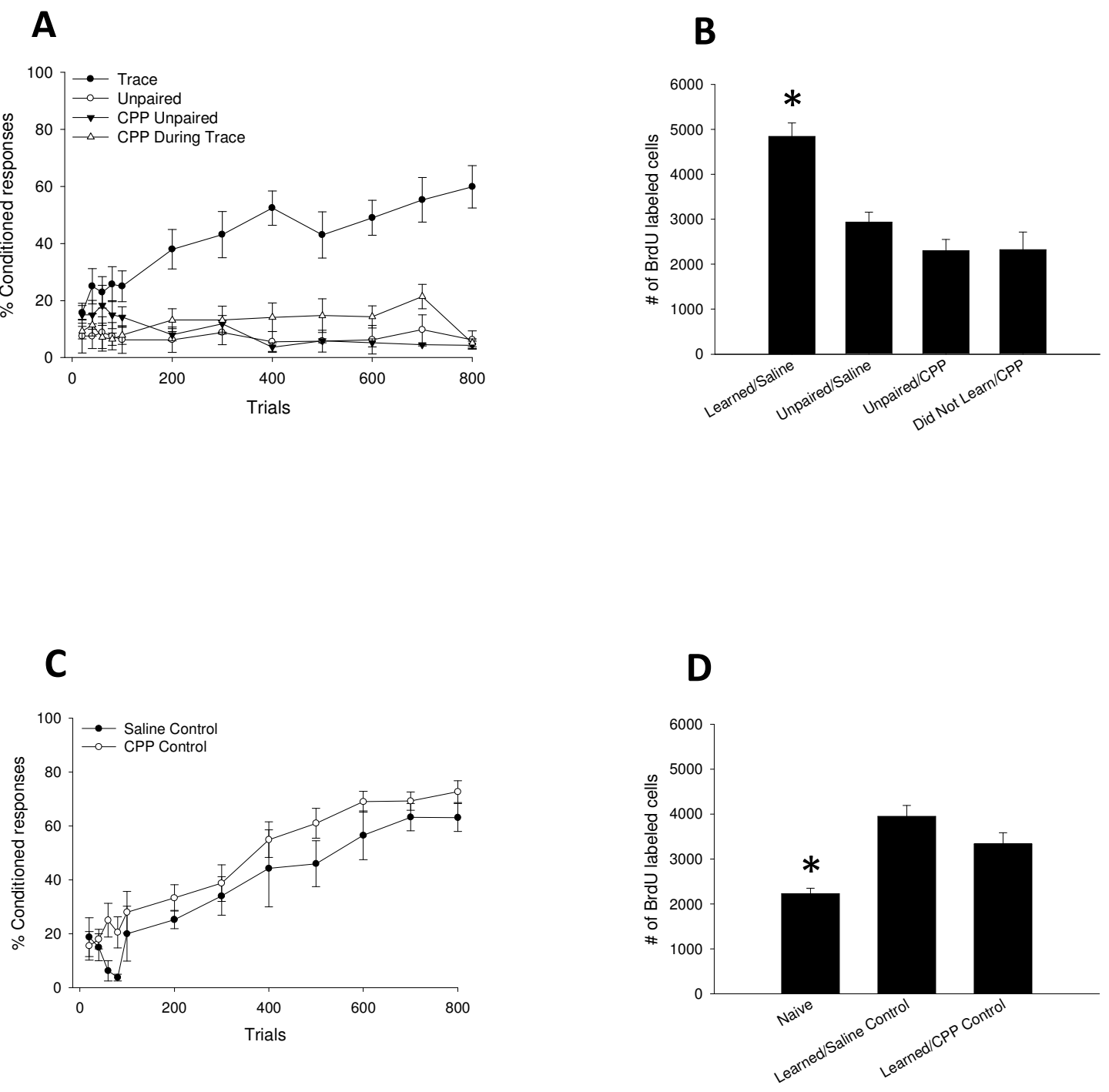
Figure 2

\section{A}

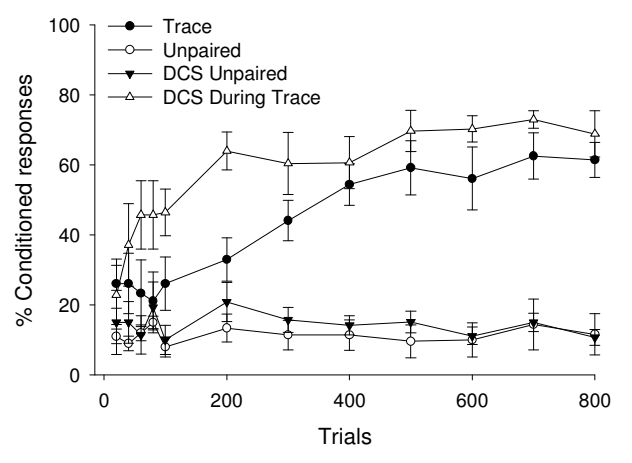

B

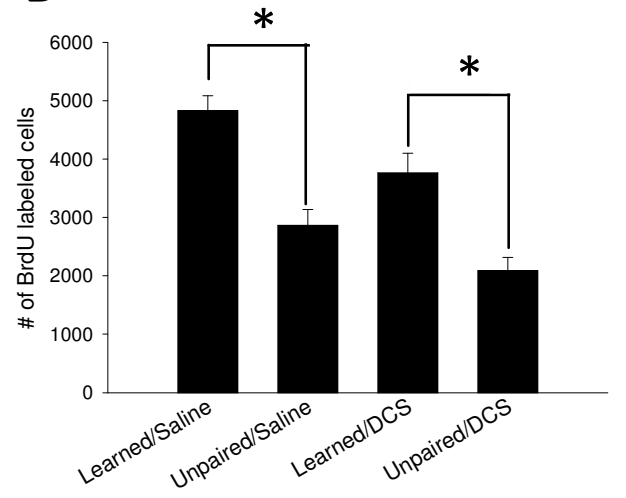

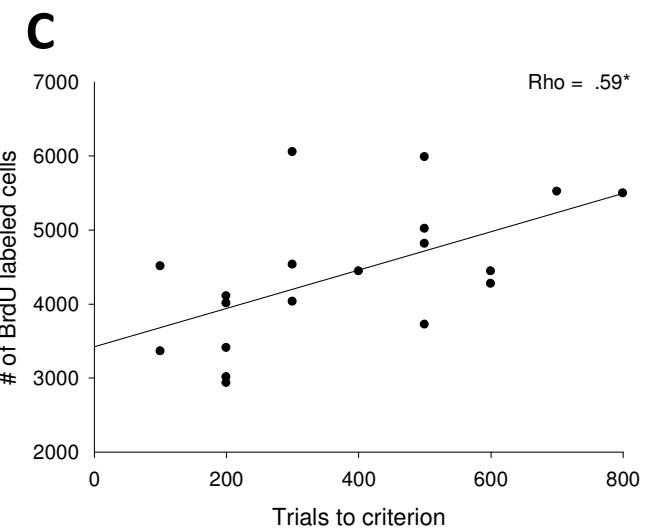


$\underline{\text { Figure } 3}$

A

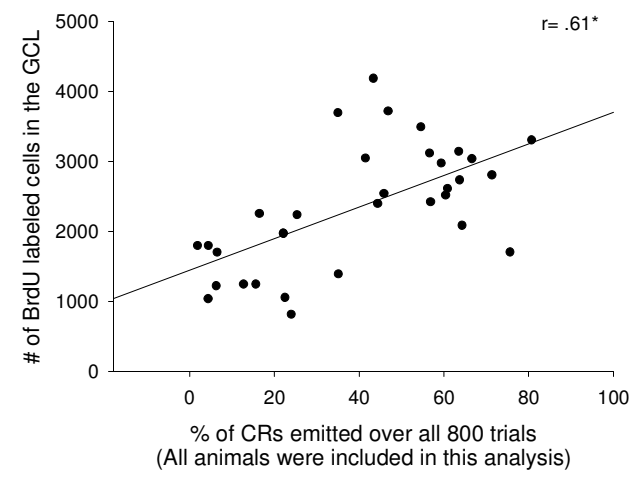

B

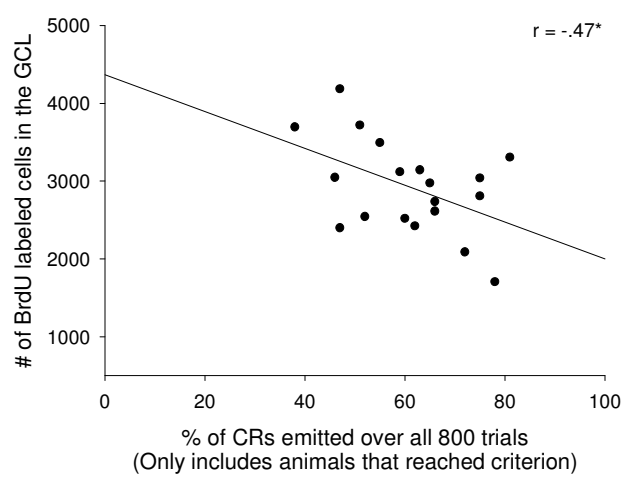


Figure 4
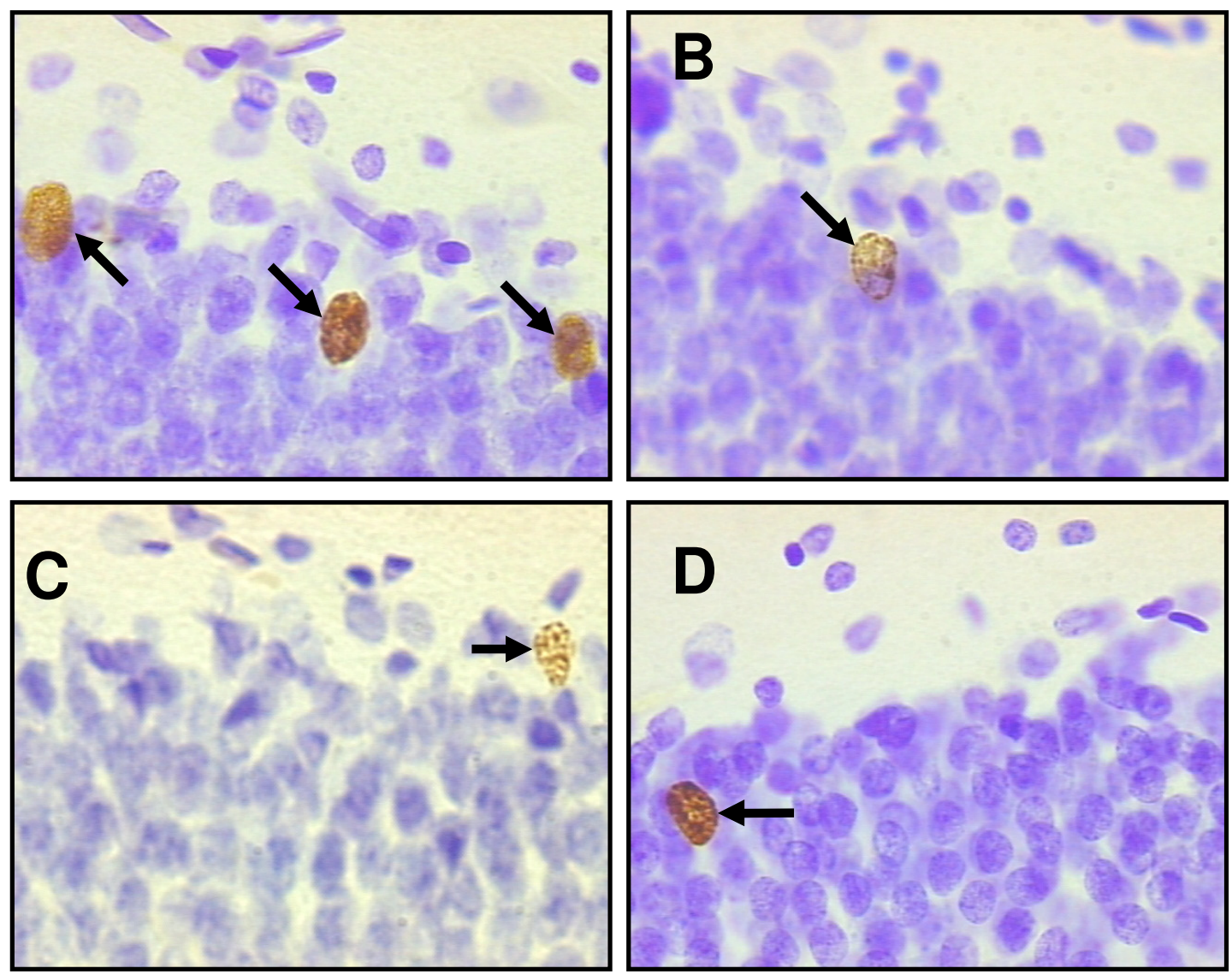


\section{$\underline{\text { Reference List }}$}

Altman,J., \& Das,G.D. (1965). Autoradiographic and histological evidence of postnatal hippocampal neurogenesis in rats. J.Comp Neurol., 124(3), 319-335.

Ambrogini,P., Cuppini,R., Cuppini,C., Ciaroni,S., Cecchini,T., Ferri,P., Sartini,S., $\&$ Del Grande,P. (2000). Spatial learning affects immature granule cell survival in adult rat dentate gyrus. Neurosci.Lett., 286(1), 21-24.

Anderson,M., Shors,T. (2010). The effects of learning on neurogenesis: Survival versus proliferation. In preparation.

Barnea,A., \& Nottebohm,F. (1994). Seasonal recruitment of hippocampal neurons in adult free-ranging black-capped chickadees. Proc.Natl.Acad.Sci.U.S.A., 91(23), 11217-11221

Cameron,H.A., Woolley,C.S., McEwen,B.S., \& Gould,E. (1993). Differentiation of newly born neurons and glia in the dentate gyrus of the adult rat. Neuroscience., 56(2), 337-344.

Cameron, H. A., McEwen, B. S., \& Gould, E. (1995). Regulation of adult neurogenesis by excitatory input and NMDA receptor activation in the dentate gyrus. J.Neurosci., 15, 4687-4692.

Cameron,H.A., \& McKay,R.D. (2001). Adult neurogenesis produces a large pool of new granule cells in the dentate gyrus. J.Comp Neurol., 435(4), 406-417.

Caviness, V.S., Jr. (1973). Time of neuron origin in the hippocampus and dentate gyrus of normal and reeler mutant mice: an autoradiographic analysis. J.Comp Neurol., 151(2), 113-120.

Dalla,C., Bangasser,D.A., Edgecomb,C., \& Shors,T.J. (2007). Neurogenesis and learning: acquisition and asymptotic performance predict how many new cells survive in the hippocampus. Neurobiol.Learn.Mem., 88(1), 143-148.

Davis, M., Ressler, K., Rothbaum, B. O., \& Richardson, R. (2006). Effects of Dcycloserine on extinction: translation from preclinical to clinical work. Biol.Psychiatry, 60, 369-375.

Dayer,A.G., Ford,A.A., Cleaver,K.M., Yassaee,M., \& Cameron,H.A. (2003). Short-term and long-term survival of new neurons in the rat dentate gyrus. J.Comp Neurol., 460(4), 563-572.

Epp, J. R., Spritzer, M. D., \& Galea, L. A. (2007). Hippocampus-dependent learning promotes survival of new neurons in the dentate gyrus at a specific time during cell maturation. Neuroscience, 149, 273-285. 
Eriksson,P.S., Perfilieva,E., Bjork-Eriksson,T., Alborn,A.M., Nordborg,C., Peterson,D.A., \& Gage,F.H. (1998). Neurogenesis in the adult human hippocampus. Nat.Med., 4(11), 1313-1317.

Gilmartin, M. R. \& McEchron, M. D. (2005). Single neurons in the dentate gyrus and CA1 of the hippocampus exhibit inverse patterns of encoding during trace fear conditioning. Behav.Neurosci., 119, 164-179.

Gould, E., Cameron, H. A., \& McEwen, B. S. (1994). Blockade of NMDA receptors increases cell death and birth in the developing rat dentate gyrus. J.Comp Neurol., 340, 551-565.

Gould,E., Reeves,A.J., Fallah,M., Tanapat,P., Gross,C.G., \& Fuchs,E. (1999b). Hippocampal neurogenesis in adult Old World primates. Proc.Natl.Acad.Sci.U.S.A., 96(9), 5263-5267.

Hastings,N.B., \& Gould,E. (1999). Rapid extension of axons into the CA3 region by adult-generated granule cells. J.Comp Neurol., 413(1), 146-154.

Henderson, G., Johnson, J. W., \& Ascher, P. (1990). Competitive antagonists and partial agonists at the glycine modulatory site of the mouse N-methyl-D-aspartate receptor. J.Physiol, 430, 189-212.

Hofmann, S. G., Meuret, A. E., Smits, J. A., Simon, N. M., Pollack, M. H., Eisenmenger, K., Shiekh, M., \& Otto, M. W. (2006). Augmentation of exposure therapy with D-cycloserine for social anxiety disorder. Arch.Gen.Psychiatry, 63, 298-304.

Hood, W. F., Compton, R. P., \& Monahan, J. B. (1989). D-cycloserine: a ligand for the N-methyl-D-aspartate coupled glycine receptor has partial agonist characteristics. Neurosci.Lett., 98, 91-95.

Kaplan,M.S., \& Hinds,J.W. (1977). Neurogenesis in the adult rat: electron microscopic analysis of light radioautographs. Science., 197(4308), 1092-1094.

Kornack,D.R., \& Rakic,P. (1999). Continuation of neurogenesis in the hippocampus of the adult macaque monkey. Proc.Natl.Acad.Sci.U.S.A., 96(10), 57685773.

Ledgerwood, L., Richardson, R., \& Cranney, J. (2003). Effects of D-cycloserine on extinction of conditioned freezing. Behav.Neurosci., 117, 341-349.

Leuner,B., Mendolia-Loffredo,S., Kozorovitskiy,Y., Samburg,D., Gould,E., \& Shors,T.J. (2004). Learning enhances the survival of new neurons beyond the time when the hippocampus is required for memory. J.Neurosci., 24(34), 7477-7481.

Leuner,B., Falduto,J., \& Shors,T.J. (2003). Associative memory formation increases the observation of dendritic spines in the hippocampus. J.Neurosci., 23(2), 659-665. 
Markakis,E.A., \& Gage,F.H. (1999). Adult-generated neurons in the dentate gyrus send axonal projections to field CA3 and are surrounded by synaptic vesicles. J.Comp Neurol.,_(4), 449-460.

Moyer, J. R., Jr., Thompson, L. T., \& Disterhoft, J. F. (1996). Trace eyeblink conditioning increases CA1 excitability in a transient and learning-specific manner. J.Neurosci., 16, 5536-5546.

Nacher, J., Rosell, D. R., Alonso-Llosa, G., \& McEwen, B. S. (2001). NMDA receptor antagonist treatment induces a long-lasting increase in the number of proliferating cells, PSA-NCAM-immunoreactive granule neurons and radial glia in the adult rat dentate gyrus. Eur.J.Neurosci., 13, 512-520.

Onur, O. A., Schlaepfer, T. E., Kukolja, J., Bauer, A., Jeung, H., Patin, A., Otte, D. M., Shah, N. J., Maier, W., Kendrick, K. M., Fink, G. R., \& Hurlemann, R. (2010). The N-Methyl-D-Aspartate Receptor Co-agonist D-Cycloserine Facilitates Declarative Learning and Hippocampal Activity in Humans. Biol.Psychiatry.

Rouaud, E. \& Billard, J. M. (2003). D-cycloserine facilitates synaptic plasticity but impairs glutamatergic neurotransmission in rat hippocampal slices.Br.J.Pharmacol., 140, 1051-1056.

Sisti,H.M., Glass,A.L., \& Shors,T.J. (2007). Neurogenesis and the spacing effect: learning over time enhances memory and the survival of new neurons. Learn.Mem., 14(5), 368-375.

Servatius,R.J., \& Shors,T.J. (1996). Early acquisition, but not retention, of the classically conditioned eyeblink response is N-methyl-D-aspartate (NMDA) receptor dependent. Behav.Neurosci., 110 (5), 1040-1048.

Shors,T.J. (2008). From stem cells to grandmother cells: how neurogenesis relates to learning and memory. Cell Stem Cell., 3(3), 253-258.

Tashiro, A., Zhao, C., \& Gage, F. H. (2006). Retrovirus-mediated single-cell gene knockout technique in adult newborn neurons in vivo. Nat.Protoc., 1, 3049-3055.

Thompson,L.T., Moskal,J.R., \& Disterhoft,J.F. (1992). Hippocampus-dependent learning facilitated by a monoclonal antibody or D-cycloserine. Nature., 359(6396), 638-641.

Thompson,L.T., \& Disterhoft,J.F. (1997). Age- and dose-dependent facilitation of associative eyeblink conditioning by D-cycloserine in rabbits. Behav.Neurosci., 111(6), 1303-1312.

Waddell,J., \& Shors,T.J. (2008). Neurogenesis, learning and associative strength. Eur.J.Neurosci., 27(11), 3020-3028. 
Waddell, J., Mallimo, E., \& Shors, T. (2010). d-cycloserine reverses the detrimental effects of stress on learning in females and enhances retention in males. Neurobiol.Learn.Mem., 93, 31-36. 